\title{
Towards a better testicular sperm extraction: novel sperm sorting technologies for non-motile sperm extracted by microdissection TESE
}

\author{
Cathy L. Mangum ${ }^{1}$, Darshan P. Patel ${ }^{1}$, Alexander R. Jafek ${ }^{2}$, Raheel Samuel ${ }^{2}$, Tim G. Jenkins ${ }^{1,3}$, \\ Kenneth I. Aston ${ }^{1,3}$, Bruce K. Gale ${ }^{2}$, James M. Hotaling ${ }^{1,3}$ \\ ${ }^{1}$ Division of Urology, Department of Surgery, University of Utah Health, Salt Lake City, UT, USA; ${ }^{2}$ Department of Mechanical Engineering, \\ ${ }^{3}$ Andrology and IVF Laboratory, Department of Surgery (Urology), University of Utah, Salt Lake City, UT, USA \\ Contributions: (I) Conception and design: CL Mangum, DP Patel, JM Hotaling; (II) Administrative support: None; (III) Provision of study materials \\ or patients: None; (IV) Collection and assembly of data: CL Mangum, DP Patel, JM Hotaling; (V) Data analysis and interpretation: CL Mangum, \\ DP Patel, JM Hotaling; (VI) Manuscript writing: All authors; (VII) Final approval of manuscript: All authors. \\ Correspondence to: James M. Hotaling, MD, MS, FECSM. Assistant Professor of Surgery (Urology), Adjunct Assistant Professor of Obstetrics/ \\ Gynecology, Division of Urology, Department of Surgery, University of Utah School of Medicine, 30 N 1900 E, Rm \# 3B420, Salt Lake City, UT \\ 84132, USA. Email: jim.hotaling@hsc.utah.edu.
}

\begin{abstract}
Non-obstructive azoospermia (NOA) is the most severe form of male factor infertility. It is characterized by a lack of spermatogenesis in the seminiferous tubules. Microdissection testicular sperm extraction (microTESE) has significantly improved testicular sperm retrieval rates compared to conventional techniques for NOA. Following testicular biopsy, the sperm is usually non-motile and contained within seminiferous tubules requiring extensive laboratory processing to find individual sperm sufficient for artificial reproductive technologies (ART). Current techniques include mechanical and enzymatic processing which is time-consuming and often damaging to sperm. We review novel techniques that may help improve sperm retrieval rates after microTESE including microfluidics (dielectrophoretic cell sorting, spiral channel sorting, and pinched flow fractionation), fluorescence-activated cell sorting (FACS), and magnetic-activated cell sorting (MACS).
\end{abstract}

Keywords: Microfluidics; sperm separation; azoospermia/therapy; male; microdissection/methods; sperm injections; intracytoplasmic/methods; sperm retrieval

Submitted Jun 14, 2019. Accepted for publication Aug 19, 2019.

doi: 10.21037/tau.2019.08.36

View this article at: http://dx.doi.org/10.21037/tau.2019.08.36

\section{Introduction}

Approximately $15 \%$ of couples fail to conceive after one year, and male factor infertility is implicated in nearly half of these cases (1). Semen analysis is the cornerstone of the male infertility workup and can provide valuable diagnostic information. Semen analysis may suggest poor sperm motility, abnormal morphology, ejaculatory tract abnormality, or inadequate sperm production. A semen sample can also be measured or quantified using parameters such as semen volume $(\mathrm{mL})$, vitality (live spermatozoa), sperm morphology, sperm concentration $\left(10^{6}\right.$ per $\left.\mathrm{mL}\right)$, and absolute sperm count (2). Further management recommendations are made based on these subjective and objective parameters of the semen analysis.

Azoospermia is characterized by the lack of sperm in the ejaculate. Azoospermia may be due to obstruction of the male reproductive tract [obstructive azoospermia $(\mathrm{OA})]$ or inadequate sperm production [non-obstructive azoospermia (NOA)]. NOA is the most severe form of male-factor infertility and is characterized by little or no sperm production in the seminiferous tubules. Up to $30-60 \%$ of males with NOA can have surgical sperm 
identified on microdissection testicular sperm extraction (microTESE) $(3,4)$. In comparison, OA is caused by ductal system obstruction leading to male factor infertility. OA is characterized by adequate testicular sperm production which may be successfully extracted through testicular sperm aspiration (TESA) or testicular sperm extraction (TESE) (5). Sperm extraction from patients with NOA is more difficult and requires additional steps before fertilization can occur using artificial reproductive technologies (ART). The introduction of intra-cytoplasmic sperm injection (ICSI) has allowed the use of even a single extracted sperm to fertilize an oocyte, which can lead to clinical pregnancy. This has offered an opportunity for men with NOA to become biological fathers $(3,6)$. Previously, severe cases of NOA required use of donor sperm to result in successful pregnancy (7). The challenge with sperm retrieval in NOA is that testicular sperm are often non-motile and extremely rare (7).

Multiple methods have been developed to extract testicular sperm in NOA. Non-selective, open biopsies have been used; however, multiple non-selective testicular biopsies may risk damage to the testes (8). TESA is a percutaneous procedure where a needle is advanced through the skin into the testicle while applying suction and the aspirated fluid is then checked for sperm. This technique carries the risk of injuring small vessels causing a hematoma and often fails to recover sperm in patients with NOA (9). In one study, fine needle aspiration with mapping (defined as more than four fine needle aspiration sites per testis following systematic planned aspiration site mapping) yielded sperm in $33 \%$ of patients with NOA (10). These sperm extraction techniques have led to TESE using a high magnification surgical microscope or microTESE. MicroTESE has higher sperm retrieval rates when compared to both random multiple-biopsy TESE and TESA for retrieving sperm in NOA (9).

In contrast to traditional TESE, microTESE is performed using a surgical microscope to distinguish dilated seminiferous tubules while minimizing tissue damage. Dissection is performed at $15-20 \times$ magnification to search for and extract large diameter tubules resulting in a selective biopsy, decreased tissue removal, and an increased yield of sperm retrieval (11). Ramasamy et al. reported $52 \%$ of men undergoing microTESE successfully had sperm retrieved and identified (12). Caroppo et al. showed that the sperm retrieval rate was higher from dilated seminiferous tubules (90\%) when compared with non-dilated tubules (7\%) (13).

Once the testicular biopsies are performed, the samples are processed to separate sperm from the biopsied tissues. Currently, sperm extraction from microTESE specimens is a labor-intensive, inefficient, and expensive process. Following surgical retrieval, the tissues are mechanically minced with needles, scissors, or angiocatheters to release sperm from the seminiferous tubules in the biopsied samples. Collagenases and other tissue degradation methods can be used to further liquefy the extracellular matrix. The resulting tissue suspension undergoes examination for sperm using an optical microscope. Generally, as they have not yet gained motility, testicular sperm is immature, and it may be difficult to distinguish it from the surrounding cells. Crabbé et al. identified 41 testicular samples from men in which no sperm was identified following mechanical mincing (14). After being exposed to erythrocyte-lysing buffer, it was possible to identify sperm in 14 of the 41 samples, which shows that sperm was contained within the seminiferous tubules before being digested using chemical processing (14).

There are several limitations to the current procedures for identifying sperm from testicular biopsies in NOA. The efficiency of sperm processing is limited by human factors, including examiner fatigue and inability to visualize sperm. Lab personnel must meticulously evaluate the testicular biopsy sample using an optical microscope which can take as many as $12-14$ hours depending on the amount of sperm present and experience level of personnel $(12,15)$. There is an inverse relationship between the time spent on searching for sperm and the likelihood of successful sperm retrieval, which ultimately impacts pregnancy rates $(12,15)$. Another concern is sperm damage during processing. Sperm is exposed to environmental and chemical processing during and after testicular extraction. Testicular tissues are often degraded by enzymatic digestion including collagenases. Collagenases are a type of matrix metalloproteinase which can dissolve tissues. Collagenase can hydrolyze the 3-dimensional helical structure of collagen to release cells from the extracellular matrix but have also been shown to digest cell surface proteins (16). This may have consequences on sperm viability for ART. A retrospective study was performed by Baukloh et al. which compared the ICSI pregnancy rate between samples which were either mechanically or enzymatically degraded (17). No differences were shown in pregnancy or live birth rates, although fertilization rates were higher in motile sperm derived from mechanical mincing versus enzyme degradation (17). In studies of nucleus pulposus cells of the vertebral disc, optimal conditions for collagenase activity increased the 
Table 1 Advantages and disadvantage of novel cell sorting technologies for non-motile cells

\begin{tabular}{|c|c|c|c|c|}
\hline Technology & Mechanism of cell sorting & Advantages & Disadvantages & Ref. \\
\hline \multirow{3}{*}{ Dielectrophoresis } & \multirow{3}{*}{$\begin{array}{l}\text { Dipole causes motion along the } \\
\text { field gradient according to the } \\
\text { intrinsic properties of the cell }\end{array}$} & Small volume manipulation & May be difficult to optimize & \multirow{3}{*}{ Gossett (18) } \\
\hline & & High percent yield feasible & $\begin{array}{l}\text { dielectrophoretic field to } \\
\text { highly heterogeneous sample }\end{array}$ & \\
\hline & & Non-labelled cells & & \\
\hline $\begin{array}{l}\text { Spiral } \\
\text { microfluidics }\end{array}$ & $\begin{array}{l}\text { Inertia of fluids in a spiral column } \\
\text { causes cells to move forward at } \\
\text { an equilibrium position for cell } \\
\text { capture based on size }\end{array}$ & Non-labelled cells & Clogging & Son (19) \\
\hline $\begin{array}{l}\text { Pinched flow } \\
\text { fractionation }\end{array}$ & $\begin{array}{l}\text { Fluid physics causes cells of } \\
\text { a certain size to move forward } \\
\text { along a stream path after aligning } \\
\text { themselves against the wall of a } \\
\text { pinched segment }\end{array}$ & Inexpensive & $\begin{array}{l}\text { Difficult to sort cells of similar } \\
\text { size }\end{array}$ & Gossett (18) \\
\hline \multirow[t]{3}{*}{ MACS } & \multirow{3}{*}{$\begin{array}{l}\text { Labelled cells stay in a magnetic- } \\
\text { activated column allowing non- } \\
\text { labelled cells to elute }\end{array}$} & Expensive & \multirow{3}{*}{$\begin{array}{l}\text { High throughput of cells } \\
\text { makes sorting highly efficient }\end{array}$} & \multirow[t]{3}{*}{ Said (20) } \\
\hline & & Low percent yield & & \\
\hline & & $\begin{array}{l}\text { Required labelling with } \\
\text { antibodies }\end{array}$ & & \\
\hline \multirow[t]{3}{*}{ FACS } & \multirow{3}{*}{$\begin{array}{l}\text { Fluorescently tagged cells can be } \\
\text { separated from non-labelled cells }\end{array}$} & Expensive & \multirow{3}{*}{$\begin{array}{l}\text { High throughput of cells } \\
\text { makes sorting highly efficient }\end{array}$} & \multirow[t]{3}{*}{ Komoda (21) } \\
\hline & & Low percent yield, & & \\
\hline & & $\begin{array}{l}\text { May not be compliant with } \\
\text { good manufacturing practice }\end{array}$ & & \\
\hline
\end{tabular}

MACS, magnetic-activated cell sorting; FACS, fluorescence-activated cell sorting.

digestion and damage to cellular tissues.

There is a strong interest in improving the processing and sorting of sperm for NOA after microTESE, which reduces the rate of human error and fatigue yet has acceptable sperm retrieval rates. In this paper, we review novel methods for sperm retrieval after microTESE including technologies with the potential to sort non-motile cells like testicular sperm.

\section{New methods for retrieving sperm}

There are several novel technologies, which may improve sperm retrieval after microTESE. These technologies include microfluidics (including dielectrophoresis and pinched-flow) that uses small streams of fluid to sort cells, magnetic-activated cell sorting (MACS), that uses a magnetically activated column of water that can sort antibody-cell surface antigen tagged cells), and fluorescenceactivated cell sorting (FACS), where fluorescent-labeled cells are sorted based on light scattering from a laser source. These methods are summarized in Table 1 . We review each of these novel technologies in further detail below.

\section{Microfluidics}

Microfluidics is the manipulation of fluids on the nanoor micro-scale for sorting. Microfluidics provides a way to bypass the deleterious effects of traditional sperm sorting. It is one of the few available sorting techniques that avoids the use of biochemical labels when sorting live cells (18). Additionally, it avoids the generation of reactive oxygen species (ROS) associated with washing and centrifugation and prolonged exposure to collagenase (22). Microfluidic processing is also efficient. Son et al. demonstrated a 
Table 2 Microfluidics devices for sorting motile and non-motile cells

\begin{tabular}{ll}
\hline Cell sorting devices relying on motility & Cell sorting devices for non-motile cells \\
\hline 1. Micro-obstacle course & 1. Dielectrophoresis cell sorting \\
2. Chemo-attraction & 2. Spiral channel inertial equilibrium cell sorting \\
3. Linear velocity/stream swim-over & 3. Pinched flow fractionation \\
4. Swimming into micro-channel & 4. Fluorescence-activated cell sorting (FACS) \\
5. Thermo-attraction & 5. Magnetic-activated cell sorting \\
\hline
\end{tabular}

5-minute sperm sample process time for leukospermic samples with a spiral microfluidic chamber which may also translate to faster processing times for microTESE samples (19).

Microfluidics is used for a wide array of applications including diagnostics, therapeutics, and cell biology and is currently used for many applications within ART (15). An early use of microfluidics for sperm sorting used microchannels that would separate healthy motile sperm from debris and non-motile cells. A modified version of this device uses chemo-attractants to coax motile sperm to swim to a specific outlet where they can be collected. Many microfluidics devices use parallel laminar streams of media and rapid temperature changes that additionally allow for sperm sorting (15). Additional modifications including flow dynamics, obstacles/barriers for the sperm, chemotaxis, and thermotaxis have been developed to enhance sperm sorting. Application of microfluidic technologies to microTESE for NOA is limited by sperm motility. However, several novel microfluidic derived technologies may be applicable for sorting non-motile cells including testicular sperm. Table 2 outlines microfluidics devices used in both motile and nonmotile cell sorting. Figure 1 illustrates each microfluidic device for cell sorting of non-motile cells.

\section{Actuated/dielectrophoresis cell sorting}

Actuated/dielectrophoresis cell sorting uses an external energy source, such as an electrical, magnetic, or acoustic field to separate non-motile cells $(23,24)$. The cells are suspended in a field gradient, inducing a dipole that can stimulate cell motility up or down the field. In this technique, two laminar streams of media are injected into the microfluidic device and run parallel to each other. The external energy source is then activated and can pull a cell towards or away from the energy field based on the size and charge to separate cells into the adjacent stream of flow, thus effectively sorting the cells from contaminants as other contaminants within the sample are not pulled into the second stream. The force exerted on a cell can be determined based on the conductivity, permittivity, and size of each cell.

de Wagenaar et al. demonstrated the ability to separate boar sperm based on cytoplasmic droplets on the flagella which is a common morphology anomaly (23). In a proof of concept experiment, they also demonstrated separation of sperm cells from $3 \mu \mathrm{m}$ beads using impedance-controlled cell sorting to show that this method could non-invasively sort sperm. As the sperm and beads passed through the microfluidic channel, they demonstrated a clear impedance change from the population of beads versus the population of sperm. When an impedance change was detected, the dielectrophoretic electrodes would activate to sort the particle into a separate channel. Future research using impedance analysis will investigate the many physical characteristics of sperm cells, which may be useful in the future of sperm-sorting (23). Ohta $e t$ al. demonstrated the utilization of opto-electric tweezers (optically induced dielectrophoresis) to non-invasively identify and sort viable live non-motile sperm from non-viable sperm without inflicting DNA damage on the cells (24). Their study was intended to show that dielectrophoresis does not injure sperm cells and used the absence of Trypan blue dye as a marker for viability. In this study, 100\% (200 individual sperm) were Trypan blue negative, and it was concluded that using this method for dielectrophoretic cell sorting maintains cell viability and could be a reliable method for separating Trypan blue cells (24). Huang et al. used dielectrophoresis charges to separate circulating tumor cells from blood based on the capacitance and conductivity of the tumor cells (25). Tumor cells have more folds on the plasma membrane when compared to other contents in the blood. These folds can increase the capacitance up to $300 \%$, allowing for dielectrophoretic manipulation of cells against a background of normal blood cells including white 


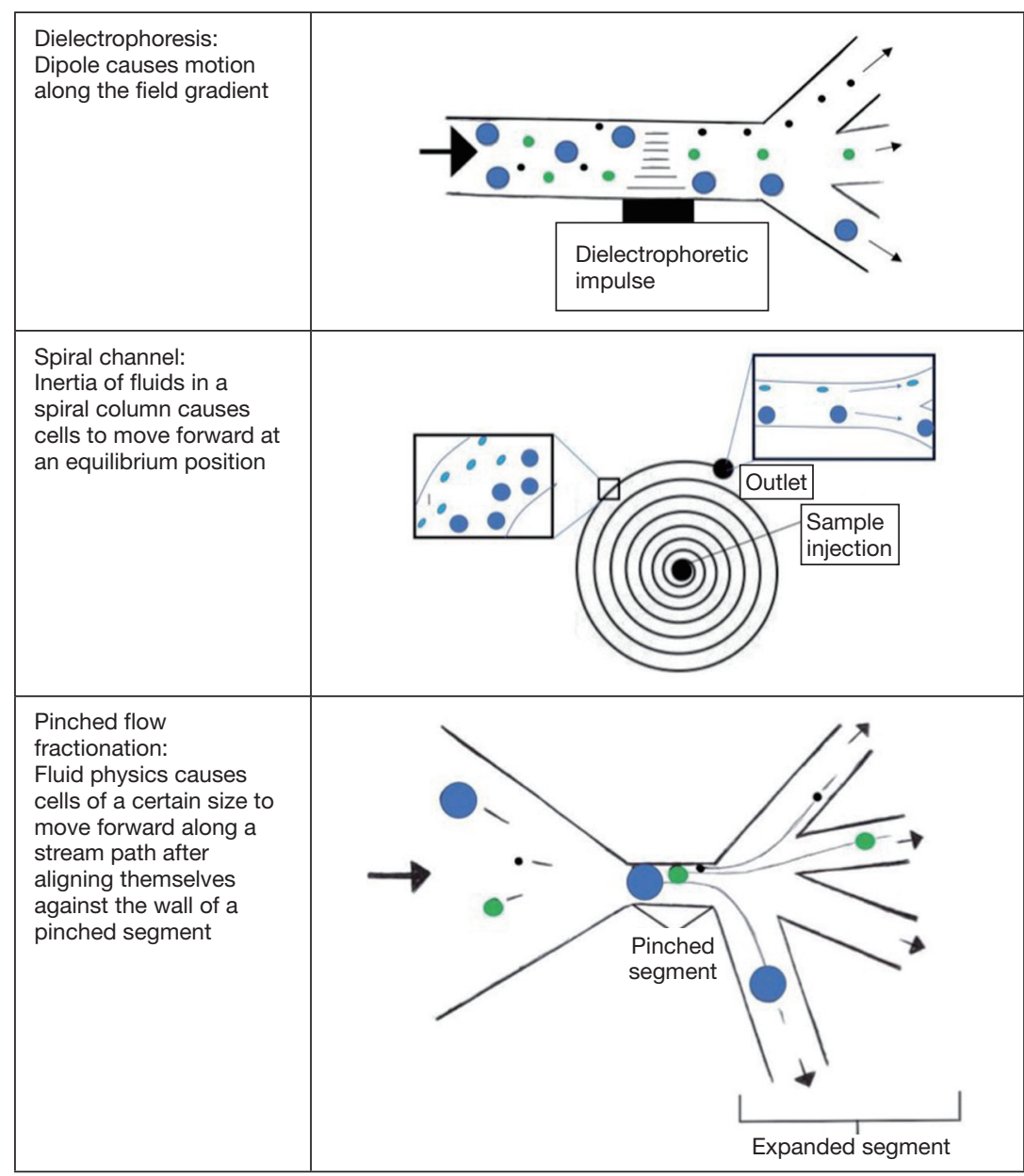

Figure 1 Illustrations of microfluidics devices for non-motile cell sorting.

blood cells which have a similar size profile as many tumor cells. This study further demonstrates that non-motile cells can be separated using laminar flow microfluidics with dielectrophoresis (25).

Although dielectrophoretic cell sorting may be a future candidate for non-motile sperm separation from a TESE sample, further research is needed in this area. There is also an incomplete understanding on how dielectrophoretic manipulation impacts sperm viability for further ART applications. Potential limitations of dielectrophoresis include identifying the optimal impedance for separation of viable sperm from cellular and extracellular debris.

\section{Spiral microfluidics}

Traditional use of microfluidics involves using parallel laminar flow through straight microchannels. One stream within the channel contains a sperm sample, and the second stream contains sperm media which flow adjacent to each other. This allows motile sperm to swim from the first stream to the second, effectively sorting motile sperm from the rest of the sample. Son $e t a l$. demonstrated a novel method for sperm separation from a simulated leukospermic sample, effectively separating sperm from other debris using inertial microfluidic theory within a spiral microfluidic channel (19). As the cells are carried forward within the spiral channel, each cell will migrate laterally until an equilibrium position is reached within the spiral. Cells will continue at this lateral position at a constant flow rate until they reach the outlet of the tube. This method bypasses the need for labeling, centrifuging, or radiolabeling and may be used for separating TESE samples. The sperm's diameter was approximated as a sphere to calculate a channel length at which an equilibrium position of sperm could be reached within the channel (19). 
Inertial microfluidic theory relies on the separation of particles in a spiral chamber based on size and shape of the constituents within a sample. The device relies on sperm size compared to the other cells in solution rather than motility, making it a viable option to separate sperm from microTESE samples. The spiraling channel dimensions were calculated based on the expected size of cellular contaminants (WBCs $12 \mu \mathrm{m}$ ) and the size of an average sperm head $(5 \mu \mathrm{m})$. It contained a single inlet with multiple outlets to separate particles at their equilibrium positions as they exited the device. For the calculation, each cell was approximated to a spherical shape. The initial study was done by separating sperm from blood cells and small debris, but with alteration this will likely be applicable for microTESE samples, which will contain a more heterogeneous sample (19). There is concern that the asymmetric shape of sperm may cause incomplete sorting or loss of viable sperm. Additionally, we have a limited understanding on how some sperm motility would impact behavior in an inertial microfluidic channel. Further refinement of the device will likely allow non-motile sperm to be separated from a wide variety of other tissue contaminants.

\section{Pinched flow fractionation}

Pinched flow fractionation separates cells based on their size as they follow a path of media flow through a pinched and then expanded micro-device. At a low Reynolds number, a particle is presumed to follow a stream of flow in a direction based on size. The Reynolds number is a dimensionless unit used within fluid mechanics to describe flow patterns. A low Reynold's number indicates laminar flow while a high Reynold's number indicates turbulent flow. The sample of cells is injected at the inlet and then the device has a "pinched" bottleneck area where particles are forced to align against the walls of the narrow segment, regardless of size, with the centers of small molecules being much closer to the walls than large molecules. Because of the differing radii between different cell types, the centers of cell types lie on different streamlines. After the pinched segment, the stream of media is allowed to expand, which amplifies the slight difference in streamlines from the pinched segment, effectively separating and sorting cells based on size as they follow their streamline. The cells can then be collected in smaller outlets. The flow of media, channel geometry, and outlet location can be altered based on the sample to control the direction of flow of particles of a particular size. This method was used by Liu et al. to separate sperm from epithelial cells for forensic analysis in sexual assault cases (26). A microfluidic channel with pinched flow separated female epithelial cells from the sperm cells based on size. They were then able to use short tandem repeats to identify male DNA fraction (94\% male) indicating a high purification rate of sperm $(15,26)$. Flow dynamics does not rely on motility, and thus may be a viable option for sperm cell sorting for TESE.

The shape of cells appears to be a very important variable within pinched flow fractionation and may limit the application for non-motile sperm. Takagi et al. observed that red blood cells, which have a disc-like shape, have disparate trajectories and poor sorting $(18,27)$. Given the highly heterogeneous mixture in a microTESE sample, it is logical that some of the larger tissue debris may clog in the pinched section of this device as well. There is also a concern that the passage of viable sperm through the pinched flow fractionation system may induce structural or mechanical damage.

\section{Magnetic-activated cell sorting (MACS)}

Another novel method for sorting sperm is the use of MACS. It is a passive sorting technique which uses antibodycoated magnetic beads which bind to antigens on the cell surface. The principal behind using MACS for sperm sorting is that damaged and early-apoptotic sperm display the phospholipid phosphatidylserine (PS) externally on the membrane. Annexin $\mathrm{V}$ is a molecule with high affinity for PS but will not bind to intact sperm (20). Within the column, microbeads conjugated with annexin $V$ are adjacent to strong magnets. As cells pass through the column, those expressing PS will bind the annexin. Cells bound to Annexin V within the column are then enriched with iron, so when placed in a strong magnetic field, they will stay on the column while intact cells will elute freely (20).

This study using MACS was performed with healthy semen samples (20). The samples were exposed to diffusion gradient centrifugation prior to MACS, which has the potential to lose sperm which could be viable for fertilization and is known to produce ROS and damage to sperm. To date, MACS has not been studied using nonmotile sperm extracted by microTESE, and may not address the separation from other live tissues within the heterogeneous sample. While MACS appears to be a viable option for selecting for sperm without membrane damage, this is limited to a relatively homogenous sperm sample. This may not function as well with microTESE samples for non-motile sperm. 


\section{Fluorescence-activated cell sorting (FACS)}

FACS is an active sorting method which requires fluorophore labeling to identify cell surface antigens for cell sorting (21). Cells must first be tagged with fluorescent antibodies or DNA labels and are subsequently placed in a stream of liquid which passes by a laser. Each cell emits light based on its size and internal structure which can scatter the cells into a collection chamber, effectively sorting nonmotile cells (21).

In a small pilot study, fluorescence activated cell sorting was used to separate non-motile sperm from microTESE samples (28). Preparation of the samples required filtration, fixation, and DNA staining with To-Pro-3, a fluorescent dye for nuclear counterstaining. The sample was then centrifuged, which has been shown in other studies to induce oxidative damage. In this pilot study, spermatozoa were successfully isolated in 4 of 5 patients, 1 of whom initially failed sperm recovery using standard tissue processing. There is a concern regarding the impact on sperm DNA fragmentation due to this sorting process, which is being actively studied by the group. Studies will need to be conducted to assess pregnancy success following sorting with FACS (28).

\section{Discussion}

Sperm retrieval from patients with NOA provides a unique challenge for assisted reproduction because the sperm is not present within the ejaculate and testicular sperm is nonmotile. MicroTESE has improved surgical sperm retrieval rates when compared with older methods but the laboratory retrieval and processing of sperm from the testicular biopsy is still time intensive and often results in a low overall sperm yield. Additionally, these processing methods may contribute to sperm damage by removing antioxidant rich seminal plasma, and induce oxidative stress and formation of free ROS (24). There is a need for new cell-sorting devices which retrieve a high yield of sperm and can avoid exposure of the sperm to labels, free radicals, and enzymatic degradation.

There are multiple merits and limitations of the cellsorting technologies which are commercially available and being investigated for non-motile sperm extraction from a heterogeneous tissue mixture after microTESE. There are several drawbacks to FACS and MACS which limit its applicability for microTESE sperm sorting. Both technologies rely on fluorophores and antibodies which can alter cell viability. Additionally, there is significant capital cost associated with these systems in addition to the costs of reagents, antibodies, and magnetic particles (18). Both methods result in high cell loss which is not acceptable for use in microTESE sperm extraction. The cell preparation includes an incubation time when labels are added and given time to conjugate which adds time to the processing of cell. Furthermore, FACS machines are difficult to clean, which can make this cell sorting device noncompliant with good manufacturing practice, which is especially problematic in cells intended for biological use in ICSI (29).

In comparison, microfluidics applications appear promising. Microfluidics technology has been utilized previously for ART; however, many of these applications rely on motile sperm. A small handful of microfluidics devices including dielectrophoretic sorting, pinched flow fractionation, and spiraled channels, are capable of sorting non-motile cells and may be applicable for sperm harvested from NOA patients. Microfluidics devices offer a labelfree technique for sorting cells by using their physical characteristics such as cell size, electrical charge, and impedance of the cells of interest (25). The creation of novel devices using microfluidic technologies can automate the process of sorting non-motile sperm and fertilizing an oocyte (30). The potential for clogging within the microchannels is the biggest limitation of microfluidics technologies. The small channel size allows for single-cell sorting, but with a heterogenous sample this may lead to clogging, which can impact sorting efficiency. Additionally, there is an incomplete understanding of how microfluidic applications impact structural and membrane integrity.

\section{Future directions}

There has been an interest in combining different technologies to improve cell sorting. Samuel et al. proposed the use of microfluidics and Raman spectroscopy to both sort and then identify viable sperm. Raman spectroscopy has recently been used in the analysis of biomolecules and may be used to identify live sperm (11). As mentioned previously, future research in the prevention of microchannel clogging will hasten the utility of microfluidic technologies for sorting non-motile sperm from microTESE samples.

\section{Conclusions}

Advances in microTESE techniques have dramatically improved rates of sperm retrieval in NOA, which can be 
used for ICSI. Despite this progress, laboratory processing and identification of viable sperm from testicular samples is daunting and time-consuming. Newer technologies in sperm sorting may increase efficiency in sperm sorting and processing for NOA. Microfluidics technologies offer a unique opportunity to improve sperm processing after microTESE for NOA.

\section{Acknowledgments}

Funding: None.

\section{Footnote}

Provenance and Peer Review: This article was commissioned by the Guest Editors (Larry I. Lipshultz, Alexander W. Pastuszak) for the focused issue "Contemporary Issues and Controversies in Men's Health" published in Translational Andrology and Urology. The article was sent for external peer review organized by the Guest Editors and the editorial office.

Conflicts of Interest: The focused issue "Contemporary Issues and Controversies in Men's Health" was commissioned by the editorial office without any funding or sponsorship. JMH serves as an unpaid editorial board member of Translational Andrology and Urology from Aug 2019 to Jul 2021. R Samuel: Nanonc-equity stake; wFluidX Inc.equity stake. TG Jenkins: Nanonc (microfluidic sperm sorting company, inherent biosciences-leadership position/ founder. KI Aston: Nanonc-equity stake. BK Gale: Nanonc-equity stake. JM Hotaling: Boston Scientific, Endo Pharmaceuticals-research and fellowship grant; Nanonc, StreamDx, Andro360, inherent biosciencesleadership position/founder. The other authors have no conflicts of interest to declare.

Ethical Statement: The authors are accountable for all aspects of the work in ensuring that questions related to the accuracy or integrity of any part of the work are appropriately investigated and resolved.

Open Access Statement: This is an Open Access article distributed in accordance with the Creative Commons Attribution-NonCommercial-NoDerivs 4.0 International License (CC BY-NC-ND 4.0), which permits the noncommercial replication and distribution of the article with the strict proviso that no changes or edits are made and the original work is properly cited (including links to both the formal publication through the relevant DOI and the license). See: https://creativecommons.org/licenses/by-nc$\mathrm{nd} / 4.0 \%$.

\section{References}

1. Agarwal A, Mulgund A, Hamada A, et al. A unique view on male infertility around the globe. Reprod Biol Endocrinol 2015;13:37.

2. Ghuman N, Ramalingam M. Male infertility. Obstet Gynaecol Reprod Med 2018;28:7-14.

3. Esteves SC. Clinical management of infertile men with nonobstructive azoospermia. Asian J Androl 2015;17:459-70.

4. Silber SJ. Microsurgical TESE and the distribution of spermatogenesis in non-obstructive azoospermia. Hum Reprod 2000;15:2278-84.

5. Craft I, Tsirigotis $M$, Bennett V, et al. Percutaneous epididymal sperm aspiration and intracytoplasmic sperm injection in the management of infertility due to obstructive azoospermia. Fertil Steril 1995;63:1038-42.

6. Vloeberghs V, Verheyen G, Haentjens P, et al. How successful is TESE-ICSI in couples with non-obstructive azoospermia? Hum Reprod 2015;30:1790-6.

7. Tournaye H. Update on surgical sperm recovery--the European view. Hum Fertil (Camb) 2010;13:242-6.

8. Shah R, Gupta C. Advances in sperm retrieval techniques in azoospermic men: A systematic review. Arab J Urol 2017;16:125-31.

9. Flannigan RK, Schlegel PN. Microdissection testicular sperm extraction: preoperative patient optimization, surgical technique, and tissue processing. Fertil Steril 2019;111:420-6.

10. Turek PJ, Cha I, Ljung BM. Systematic fine-needle aspiration of the testis: correlation to biopsy and results of organ "mapping" for mature sperm in azoospermic men. Urology 1997;49:743-8.

11. Samuel R, Badamjav O, Murphy KE, et al. Microfluidics: The future of microdissection TESE? Syst Biol Reprod Med 2016;62:161-70.

12. Ramasamy R, Fisher ES, Ricci JA, et al. Duration of microdissection testicular sperm extraction procedures: relationship to sperm retrieval success. J Urol 2011;185:1394-7.

13. Caroppo E, Colpi EM, Gazzano G, et al. The seminiferous tubule caliber pattern as evaluated at high magnification during microdissection testicular sperm extraction 
predicts sperm retrieval in patients with non-obstructive azoospermia. Andrology 2019;7:8-14.

14. Crabbé E, Verheyen G, Silber S, et al. Enzymatic digestion of testicular tissue may rescue the intracytoplasmic sperm injection cycle in some patients with non-obstructive azoospermia. Hum Reprod 1998;13:2791-6.

15. Samuel R, Feng H, Jafek A, et al. Microfluidic-based sperm sorting \& analysis for treatment of male infertility. Transl Androl Urol 2018;7:S336-47.

16. Feng X, Liu L, Yu BQ, et al. Effect of optimized collagenase digestion on isolated and cultured nucleus pulposus cells in degenerated intervertebral discs. Medicine (Baltimore) 2018;97:e12977.

17. Baukloh V, German Society for Human Reproductive Biology. Retrospective multicentre study on mechanical and enzymatic preparation of fresh and cryopreserved testicular biopsies. Hum Reprod 2002;17:1788-94.

18. Gossett DR, Weaver WM, Mach AJ, et al. Label-free cell separation and sorting in microfluidic systems. Anal Bioanal Chem 2010;397:3249-67.

19. Son J, Samuel R, Gale BK, et al. Separation of sperm cells from samples containing high concentrations of white blood cells using a spiral channel. Biomicrofluidics 2017;11:054106.

20. Said TM, Agarwal A, Grunewald S, et al. Evaluation of sperm recovery following annexin $\mathrm{V}$ magneticactivated cell sorting separation. Reprod Biomed Online 2006;13:336-9.

21. Komoda T, Matsunaga T. Chapter 6 - Biotechnological Study. In: Komoda T, Matsunaga T. editors. Biochemistry for Medical Professionals. Boston: Academic Press; 2015:75-92.

Cite this article as: Mangum CL, Patel DP, Jafek AR, Samuel R, Jenkins TG, Aston KI, Gale BK, Hotaling JM. Towards a better testicular sperm extraction: novel sperm sorting technologies for non-motile sperm extracted by microdissection TESE. Transl Androl Urol 2020;9(Suppl 2):S206-S214. doi: 10.21037/tau.2019.08.36
22. Rappa KL, Rodriguez HF, Hakkarainen GC, et al. Sperm processing for advanced reproductive technologies: Where are we today? Biotechnol Adv 2016;34:578-87.

23. de Wagenaar B, Dekker S, de Boer HL, et al. Towards microfluidic sperm refinement: impedance-based analysis and sorting of sperm cells. Lab Chip 2016;16:1514-22.

24. Ohta AT, Garcia M, Valley JK, et al. Motile and nonmotile sperm diagnostic manipulation using optoelectronic tweezers. Lab Chip 2010;10:3213-7.

25. Huang SB, Wu MH, Lin YH, et al. High-purity and label-free isolation of circulating tumor cells (CTCs) in a microfluidic platform by using opticallyinduced-dielectrophoretic (ODEP) force. Lab Chip 2013;13:1371-83.

26. Liu W, Chen W, Liu R, et al. Separation of sperm and epithelial cells based on the hydrodynamic effect for forensic analysis. Biomicrofluidics 2015;9:044127.

27. Takagi J, Yamada M, Yasuda M, et al. Continuous particle separation in a microchannel having asymmetrically arranged multiple branches. Lab Chip 2005;5:778-84.

28. Mittal S, Mielnik A, Bolyakov A, et al. Pd68-01 Pilot Study Results Using Fluorescence Activated Cell Sorting of Spermatozoa from Testis Tissue: A Novel Method for Sperm Isolation after TESE. J Urol 2017;197.

29. Faraghat SA, Hoettges KF, Steinbach MK, et al. High-throughput, low-loss, low-cost, and label-free cell separation using electrophysiology-activated cell enrichment. Proc Natl Acad Sci U S A 2017;114:4591-6.

30. Ma R, Xie L, Han C, et al. In vitro fertilization on a single-oocyte positioning system integrated with motile sperm selection and early embryo development. Anal Chem 2011;83:2964-70. 